\title{
On the Rovibrational Partition Function of Molecular Hydrogen at High Temperatures
}

\author{
Antonio Riganelli, Frederico V. Prudente, and António J. C. Varandas* \\ Departamento de Química, Universidade de Coimbra, P-3049 Coimbra Codex, Portugal
}

Received: April 10, 2001; In Final Form: June 18, 2001

We report a comparative study of the vibrational and rovibrational partition functions using several quantum and classical statistical mechanics approaches. The calculations refer to $\mathrm{H}_{2}$, but the conclusions are anticipated to be valid also for larger systems.

\section{Introduction}

The partition function is an essential construct to interpret the macroscopic world in terms of the properties of atoms and molecules. Its crucial role is indeed well-established both in thermodynamics and chemical kinetics, as shown by the considerable effort involved in developing accurate methods for its calculation. ${ }^{1,2}$

In quantum statistical mechanics, the canonical partition function assumes the form

$$
\mathcal{Q}=\sum_{j} \exp \left(-\beta E_{j}\right)
$$

where $\beta=1 / k T, T$ is the temperature, $E_{j}$ is the energy eigenvalue associated to quantum state $j$, and $k$ is the Boltzmann constant. For an ideal gas made of $N$ identical molecules, the BornOppenheimer approximation leads to ${ }^{3,4}$

$$
\mathcal{2}=\frac{1}{(N) !}\left(q_{\mathrm{t}} q_{\mathrm{e}} q_{\mathrm{vr}}\right)^{N}
$$

where $q_{\mathrm{t}}, q_{\mathrm{e}}$, and $q_{\mathrm{vr}}$ are the translational, electronic and rovibrational partition functions for an individual molecule. Although $q_{\mathrm{t}}$ can be calculated using the perfect gas formalism and $q_{\mathrm{e}}$ is unity if no electronic excited states are involved, $q_{\mathrm{vr}}$ assumes the sum-over-states form

$$
q_{\mathrm{vr}}(T)=\sum_{i} g_{i} \exp \left(-\beta E_{i}\right)
$$

where $E_{i}$ denotes now a bound molecular rovibrational state and $g_{i}$ is the corresponding degeneracy factor. To use eq 3 , one then requires the complete rovibrational spectra of the molecule, which limits the applicability of such an approach to small molecules (notable examples are applications to the benchmark systems $\mathrm{H}_{3}{ }^{+5}$ and $\mathrm{H}_{2} \mathrm{O}^{6}$ ).

An alternative and appealing approach to the calculation of $q_{\mathrm{vr}}$ comes from Feynman's path integral (FPI) formulation of quantum statistical mechanics. ${ }^{7,8}$ According to such a formalism, the partition function assumes the form

$$
q_{\mathrm{vr}}(T)=\int \mathrm{d} \mathbf{x} \int_{\mathbf{x}}^{\mathbf{x}} \mathscr{D} \mathbf{x}(s) \exp \{-S[\mathbf{x}(s), \dot{\mathbf{x}}(s)]\}
$$

* To whom correspondence should be addressed. E-mail: varandas@ qtvs1.qui.uc.pt. where

$$
S[\mathbf{x}(s), \dot{\mathbf{x}}(s)]=\int_{0}^{\beta \hbar} H[\mathbf{x}(s), \dot{\mathbf{x}}(s)] \mathrm{d} s
$$

is the action of the classical energy functional corresponding to the path $\mathbf{x}(s)$ in imaginary (thermodynamic) time $s=$ it, and the notation $\int_{\mathbf{x}}^{\mathbf{x}} \mathscr{D} \mathbf{x}(s)[\cdots]$ implies that integration over all paths is constrained to begin and end at the same point $\mathbf{x}$. Using Trotter's formula, ${ }^{9}$ the discrete path integral (DPI) representation of eq 4 assumes the form ${ }^{1,10}$

$$
\begin{aligned}
& q_{\mathrm{vr}}(T)=\left[\prod_{j=1}^{n}\left(\frac{\mu P}{2 \pi \beta \hbar^{2}}\right)^{P / 2}\right] \int \mathrm{d} \mathbf{x}^{1} \cdots \mathrm{d} \mathbf{x}^{P} \times \\
& \quad \exp \left\{-\beta\left[\frac{\mu P}{2 \hbar^{2} \beta^{2}} \sum_{i=1}^{P}\left|\mathbf{x}^{i+1}-\mathbf{x}^{i}\right|^{2}+\frac{1}{2 P} \sum_{i=1}^{P}\left(V\left(\mathbf{x}^{i}\right)+V\left(\mathbf{x}^{i+1}\right)\right)\right]\right\}
\end{aligned}
$$

where $\hbar$ is the Planck constant divided by $2 \pi, P$ is the number of discretizations of $s, n$ is the number of degrees of freedom, $\mu$ is the reduced mass, $V\left(\mathbf{x}^{i}\right)$ is the potential energy calculated at point $\mathbf{x}^{i}$, and $\mathbf{x}^{P+1}=\mathbf{x}^{1}$. It is important to point out that the expression for the potential terms in eq 6 is not unique, although all forms lead in principle to the same result. ${ }^{11}$ Chandler and Woolynes $^{12}$ have shown that there is a strict isomorphism between the DPI representation and classical statistical mechanics; thus, within this context, the DPI calculation can be regarded as a classical one. Actually, the use of the FPI formulation associated with Monte Carlo techniques has experienced enormous advances in computational thermodynamics (see ref 1 and references therein).

In classical statistical mechanics, the sum-over-states is replaced by the corresponding multidimensional phase space integral. Thus, the classical rovibrational partition function assumes the form

$$
q_{\mathrm{vr}}(T)=\frac{1}{h^{n}} \int \cdots \int \exp \{-\beta H(\mathbf{q}, \mathbf{p})\} \mathrm{d} \mathbf{q} \mathrm{d} \mathbf{p}
$$

where $H(\mathbf{q}, \mathbf{p})$ is the classical Hamiltonian, $\mathbf{q}$ is the vector of generalized coordinates, and $\mathbf{p}$ is the vector of conjugate momenta; we will assume as reference throughout this work the energy of the minimum of the potential energy surface (curve). Note that in some situations ${ }^{13-16}$ the classical picture gives results accurate enough to make quantum calculations 
unnecessary, whereas in others (for which the sum over states is still computationally out of reach), one can think of coping with the breakdown of classical statistical mechanics (especially at low temperatures) by using quantum corrections. For recent work which discusses how to account for the deviation of the classical rovibrational partition function from the exact quantum result, the reader is referred to refs $17-19$.

Frequently, the integration over momenta in eq 7 can be carried out as a simple Gaussian integral, i.e., by integrating explicitly the momenta between $-\infty$ and $+\infty$. In this case, the rovibrational partition function factorizes as the product of a term depending only on the temperature by the classical configurational integral ${ }^{3,20}$

$$
q_{\mathrm{vr}}(T)=\frac{1}{\lambda^{n}} \int \exp \{-\beta V(\mathbf{q})\} \mathrm{d} \mathbf{q}
$$

where $\lambda=\left(\beta h^{2} / 2 \pi \mu\right)^{1 / 2}$ is the thermal wavelength. For diatomic molecules, if only vibrational motion is considered, one has $n=1$ and eq 8 yields

$$
q_{\mathrm{v}}(T)=\frac{1}{\lambda} \int_{0}^{\infty} \exp \{-\beta V(r)\} \mathrm{d} r
$$

with $r$ being the internuclear distance. However, if rovibrational motion is considered, $n=3$ and the integration over the polar angles $\theta$ and $\phi$ leads to

$$
q_{\mathrm{vr}}(T)=\frac{4 \pi}{\lambda^{3}} \int_{0}^{\infty} \exp \{-\beta V(r)\} r^{2} \mathrm{~d} r
$$

Note that the usual textbook derivation (eq 8) is formally correct for systems having an infinite number of bound states. This is the case for potential functions which do not assume a finite value at large distances (e.g., harmonic or quasiharmonic models) and when molecular motion is confined to a finite volume. An example of use of eq 8 is the calculation of the equilibrium constant for reactions of the type $\mathrm{X}+\mathrm{C}_{60} \rightleftharpoons \mathrm{X} @ \mathrm{C}_{60}$ where $\mathrm{X}$ is an halogen atom trapped inside the fullerene cage (e.g., ref 21).

However, for systems having a finite number of discrete quantum states (such as those satisfying the condition $\lim _{\mathbf{q} \rightarrow \infty}$ $V(\mathbf{q}) \neq \infty)$, the use of the configurational integral fails at high temperatures. The main reason for such a failure is the fact that integration over momenta to obtain eq 8 implicitly considers the molecule to have an infinite number of states. In other words, one includes contributions from dissociated species which are formally divergent as they scale linearly with the volume of the system. This feature arises also in the FPI formulation, where an integration over momenta is performed as simple Gaussian integrals to obtain eq 6 (see Chapter 1 of ref 11). In fact, Schenter ${ }^{22}$ has recently discussed the same problem in the study of the enthalpy of formation of the water dimer, whereas Truhlar and co-workers ${ }^{2,23}$ have pointed out such a feature in calculations of molecular rovibrational partition functions employing the Fourier path integral Monte Carlo method.

To overcome the above problem (common to both classical and FPI formulations), it is necessary to limit the involved integrations to regions which correspond to bound-state situations. Although this is an open problem within the FPI framework, ${ }^{22,23}$ in classical statistical mechanics, it can be taken into account by requiring that the hypervolume of integration in eq 7 is limited to phase space regions for which the internal energy is smaller than the dissociation energy, $0 \leq H(\mathbf{q}, \mathbf{p}) \leq$ $E_{\mathrm{d}}$. In general, for a polyatomic molecule, calculations of the phase space integral in eq 7 require the use of a numerical technique. However, for diatomic molecules, the integration over momenta can be done analytically by considering such restrictions on the energy. For the vibrational partition function, eq 7 gives

$$
\begin{aligned}
q_{\mathrm{v}}(T)= & \left(\frac{2 \mu k T}{h^{2}}\right)^{1 / 2} \exp \left(\beta E_{\mathrm{d}}\right) \int_{\sigma}^{\infty} \times \\
& \exp \left\{-\beta V^{\prime}(r)\right\}\left[2 \int_{0}^{\sqrt{ }\left(-\beta \mathrm{V}^{\prime}(r)\right)} \exp \left(-p^{2}\right) \mathrm{d} p\right] \mathrm{d} r \\
= & \frac{1}{\lambda} \exp \left(\beta E_{\mathrm{d}}\right) \int_{\sigma}^{\infty} \exp \left\{-\beta V^{\prime}(r)\right\} \operatorname{erf}\left[-\beta V^{\prime}(r)\right]^{1 / 2} \mathrm{~d} r
\end{aligned}
$$

whereas for the rovibrational partition function, one has ${ }^{13,24}$

$$
\begin{aligned}
& q_{\mathrm{vr}}(T)=\frac{8 \sqrt{\pi}}{\lambda^{3}} \exp \left(\beta E_{\mathrm{d}}\right) \int_{\sigma}^{\infty} \exp \left\{-\beta V^{\prime}(r)\right\} \times \\
& \left\{\frac{\sqrt{\pi}}{2} \operatorname{erf}\left[-\beta V^{\prime}(r)\right]^{1 / 2}-\left[-\beta V^{\prime}(r)\right]^{1 / 2} \exp \left\{\beta V^{\prime}(r)\right\}\right\} r^{2} \mathrm{~d} r
\end{aligned}
$$

where $V^{\prime}(r)=V(r)-E_{\mathrm{d}}$, $\operatorname{erf}(\cdots)$ is the error function, ${ }^{25}$ and $\sigma$ is the internuclear distance at which $V^{\prime}(r)=0$.

The major goal of this work is to discuss the implications of using the FPI quantum formulation in eq 6 and the classical approaches in eqs $9-12$ to calculate the vibrational and rovibrational partition functions. Thus, we hope to shed some light on a simple but often overlooked question. As an example, we have chosen the $\mathrm{H}_{2}$ molecule. A strong motivation for this selection relates to the importance of the molecular hydrogen high-temperature thermodynamics in astrophysics. ${ }^{18,26}$ Thus, we compare the results reported in this work with Irwin's ${ }^{26}$ accurate polynomial fit of $q_{\mathrm{vr}}$ to spectroscopic data. The plan of the paper is as follows. Section 2 gives the details of the calculations, where the results of $q_{\mathrm{v}}$ and $q_{\mathrm{vr}}$ are also discussed. Some conclusions are in section 3 .

\section{Results and Discussion}

The $\mathrm{H}_{2}$ potential energy curve is based on the EHFACE2U model. ${ }^{27,28}$ Using this curve, we have calculated the exact quantum mechanical vibrational partition function in eq 3 and the quantum DPI formula in eq 6 for $n=1$. Moreover, we have computed the classical partition functions in eqs 9 and 11. The details concerning the various calculations are given next.

We begin with the calculation of the vibrational spectrum $\left\{E_{i}\right\}$. For this, we have employed the discrete variable representation (DVR) method ${ }^{29-31}$ as proposed by Colbert and Miller, ${ }^{29}$ using a grid of $N=500$ equally spaced points over the integration interval $(a, b)=\left(0.0,25.0 a_{0}\right)$. The calculated levels were then used to carry out the sum-over-states in eq 3 up to the last bound state supported by the potential energy curve (the outer turning point referring to this state occurs at $\left.r=6.0661 a_{0}\right)$.

To calculate the DPI integral in eq 6 (with $n=1$ ), we have employed the transfer matrix grid method by following the procedure described in refs 32 and 33 . The values of the parameters [see eqs 2.6-2.7 of ref 32] used to perform such calculation are $\Delta x=0.025 a_{0}$, and $N=599$, corresponding to a radial integration interval of $(a, b)=\left(0.0,15.0 a_{0}\right)$. The parameter $P$ has been optimized at each temperature until convergence has been reached. Such an optimization was done by varying $P$ until $q_{\mathrm{v}}$ reached its minimum value at each temperature. In fact, such a value should provide an upper bound 


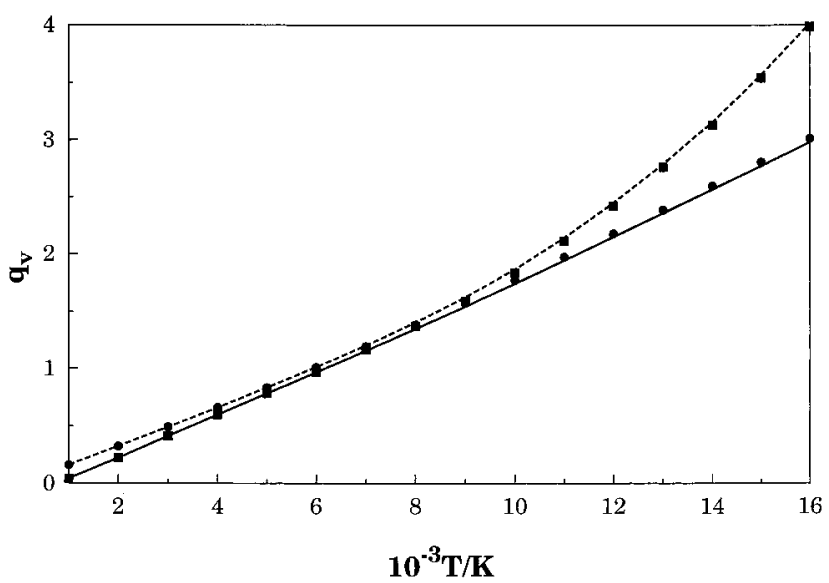

Figure 1. Vibrational partition function of $\mathrm{H}_{2}$ molecule: ( - ) quantum results, eq 3; (- - ) classical results with no restrictions to bound states as in eq 9 ; $(\bullet)$ classical results with restrictions to bound states as in eq $11 ;(\boldsymbol{\square})$ FPI results, eq 6.

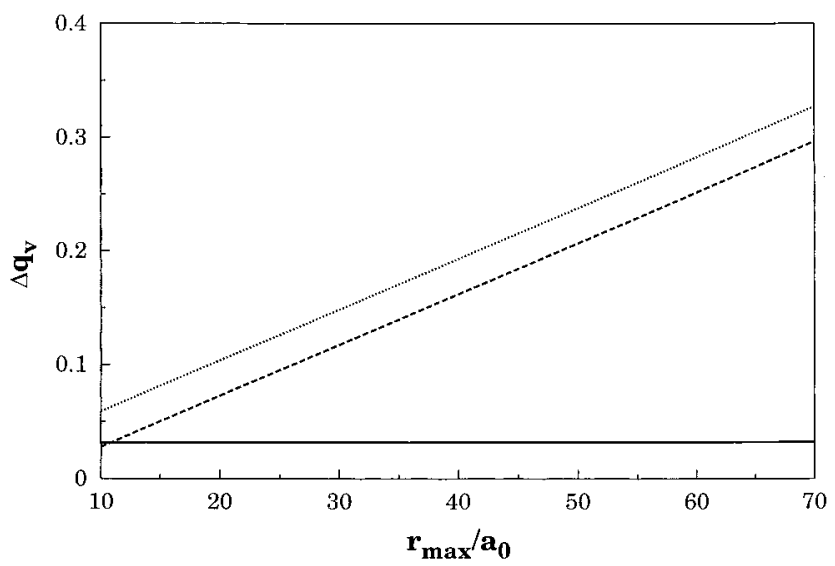

Figure 2. Error relative to the quantum results as a function of the interatomic distance $(r)$ for $q_{\mathrm{v}}$ at $T=9000 \mathrm{~K}$ : ( -) classical results with restrictions to bound states as in eq $11 ;(\cdots)$ classical results with no restrictions to bound states as in eq 9; (- -) FPI results, eq 6.

to the exact result, because this is in principle obtained for infinitesimal steps of the imaginary time $(P \rightarrow \infty)$, whereas numerical truncation errors are expected to be positive [because of appearing as the argument of the exponential in eq 6] and increase after the step size reaches some minimum value.

The classical calculations referring to eqs 9 and 11 were carried out using a standard numerical integrator, with the integration limits fixed at $r_{\max }=15.0 a_{0}$ and $\sigma=0.7760 a_{0}$.

The values of $q_{\mathrm{v}}$ over the range of temperatures of astrophysical interest ${ }^{26}$ are shown in Figure 1. Clearly, the classical $q_{\mathrm{v}}$ based on eq 11 is seen to converge to the quantum result with increasing temperature. Conversely, the classical vibrational partition function from eq 9 is shown to diverge with temperature. Figure 1 further shows that the FPI formulation gives accurate results at low and moderate temperatures but diverges at high ones $(T \geq 7000 \mathrm{~K})$ where the classical partition function calculated using eq 9 also diverges from the accurate classical counterpart. This can be attributed to the fact that both formulations consider the dissociated molecule as active species which contribute to the partition function. This remark is further exploited in Figure 2 by plotting, as a function of the upper limit of integration $\left(r_{\max }\right)$ for $T=9000 \mathrm{~K}$, the error relative to the sum-over-states result obtained by using eqs 6,9 , and 11 . To correctly apply the transfer matrix grid method of DPI, the calculations for the various $r_{\max }$ values were carried out by fixing $\Delta x$ and the optimum $P$ value while varying $N$. Clearly, the errors

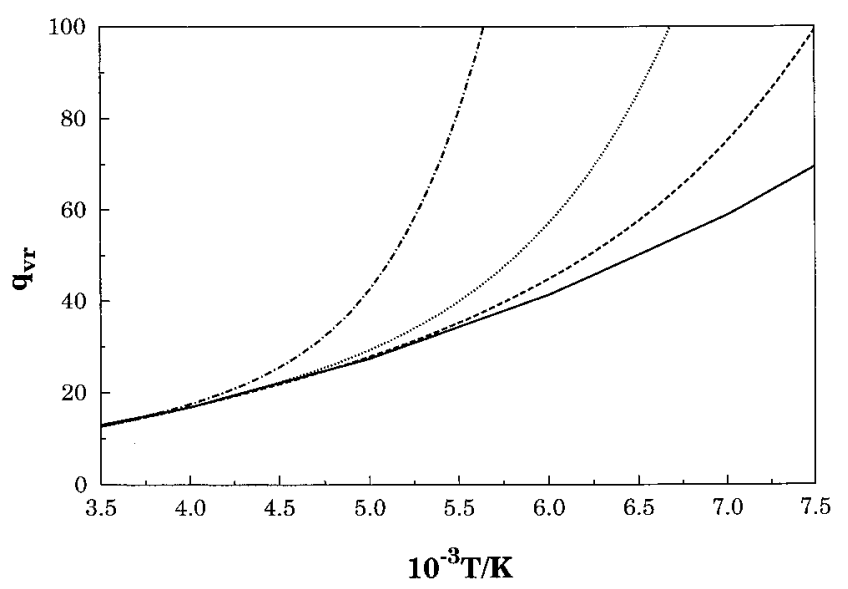

Figure 3. Classical rovibrational partition function of $\mathrm{H}_{2}$ molecule: (-) eq $12 ;(--)$ eq 10 with $r_{\max }=15.0 a_{0} ;(\cdots)$ eq 10 with $r_{\max }=$ $25.0 a_{0} ;\left(-\bullet^{-}\right)$eq 10 with $r_{\max }=50.0 a_{0}$. Except for the results shown by the solid line, all calculations are with no restrictions to bound states.

in the classical configurational integral of eq 9 and quantum DPI method of eq 6 scale linearly with $r_{\max }$. However, the analysis shows that the error does not depend on $r_{\max }$ when evaluating the classical partition function using eq 11. Thus, eq 6 can be regarded as a very good approximation for lowtemperature regimes but cannot reproduce the correct results at high temperatures. We conclude this paragraph by noting that we also carried exploratory calculations using the simple method proposed by Mielke et al. ${ }^{23}$ to avoid consideration of dissociated species within the FPI formulation. Their method consists of neglecting all paths for which the energy of the configuration space sample point, $\mathbf{x}$, is above the energy for dissociation. In fact, following their own suggestion, ${ }^{23}$ we have instead neglected contributions from paths where the potential energy at $\mathbf{x}$ is above the dissociation limit. Our test calculations have shown little or no improvement specially at high temperatures, which is probably due to having ignored the kinetic energy in such a criterion.

We now address the calculation of the rovibrational partition function. Figure 3 plots the classical $q_{\mathrm{vr}}$ calculated from eqs 10 and 12 over the temperature range $(3500 \leq T \leq 7500 \mathrm{~K})$. The various broken lines refer to distinct values of the upper integration limit $\left(r_{\max }=15.0,25.0,50.0 a_{0}\right)$ in eq 10 , whereas the solid curve refers to eq 12 using $r_{\max }=25.0 a_{0}$. It is seen that, for a given temperature, the point of divergence from the correct classical curve (shown by the solid line) is a function of the upper integration limit: it occurs at increasingly higher temperatures with decreasing $r_{\max }$. In fact, as the potential is not bound for $r \rightarrow \infty$, the available volume approaches infinity as $r_{\max } \rightarrow \infty$.

Finally, Table 1 compares the various calculated $q_{\mathrm{vr}}$ with the best available estimates ${ }^{26}$ calculated from spectroscopic data. These correspond to Irwin's fitting formula ${ }^{26}$

$$
\ln q_{\mathrm{vr}}(T)=\sum_{i=1}^{8} A_{i}(\ln T)^{i}
$$

where $1000 \leq T / K \leq 16000$ and the coefficients $A_{i}$ are adjustable parameters. ${ }^{26}$ Note that Irwin's values were calculated by taking as reference the zero-point energy of the system $\left(E_{0}=2167.1794 \mathrm{~cm}^{-1}\right)$. Thus, his results have been multiplied by $\exp \left(-\beta E_{0}\right)$. Because of spin statistics, the sum-over-states has been weighted using the proper nuclear spin factor. ${ }^{3,26}$ In turn, the classical $q_{\mathrm{vr}}$ has been calculated from eq 12 using $r_{\max }=25.0 a_{0}$, and then multiplied by $1 / 2$ to account for the 
TABLE 1: Rovibrational Partition Function for $\mathbf{H}_{2}$ Molecule Calculated with Different Methods: (A) Quantum Results [eq 3], This Work; (B) Classical Results [eq 12], This Work; (C) Irwin Results ${ }^{26}$

\begin{tabular}{cccc}
\hline & \multicolumn{3}{c}{$\mathrm{q}_{\mathrm{vr}}{ }^{\mathrm{a}}$} \\
\cline { 2 - 4 }$T / \mathrm{K}$ & \multicolumn{1}{c}{$\mathrm{A}$} & \multicolumn{1}{c}{$\mathrm{B}$} & $\mathrm{C}$ \\
\hline 1000.0 & 0.272 & 0.940 & 0.272 \\
2000.0 & 2.741 & 3.898 & 2.737 \\
3000.0 & 7.783 & 9.108 & 7.768 \\
4000.0 & 15.456 & 16.851 & 15.423 \\
5000.0 & 26.043 & 27.471 & 26.001 \\
6000.0 & 39.907 & 41.350 & 39.916 \\
7000.0 & 57.376 & 58.826 & 57.604 \\
8000.0 & 78.640 & 80.089 & 79.424 \\
9000.0 & 103.696 & 105.140 & 105.567 \\
10000.0 & 132.368 & 133.803 & 135.948 \\
11000.0 & 164.343 & 165.765 & 170.127 \\
12000.0 & 199.228 & 200.635 & 207.248 \\
13000.0 & 236.591 & 237.980 & 246.026 \\
14000.0 & 275.996 & 277.367 & 284.788 \\
15000.0 & 317.029 & 318.379 & 321.575 \\
16000.0 & 359.305 & 360.634 & 354.299
\end{tabular}

${ }^{a}$ The calculations are performed with the zero of energy at the minimum of the potential energy curve.

nuclear symmetry. Clearly, Table 1 shows that the classical partition function tends to the quantum one as the temperature increases but shows serious discrepancies for temperatures below $T=5000 \mathrm{~K}$. This is an expected result which can in principle be ameliorated by using quantum corrections. ${ }^{19}$ Moreover, the spectroscopic $q_{\mathrm{vr}}$ values are shown to be accurate within $1 \%$ or so when compared with the exact quantum ones up to $T=8000$ $\mathrm{K}$. From this temperature upward, the classical calculations based on eq 10 show a better accord with the exact quantum results, with the error in the last tabulated temperature being only $0.4 \%$ (cf. $1.8 \%$ for Irwin's formula).

\section{Conclusions}

We have reported calculations of the vibrational and rovibrational partition functions for the $\mathrm{H}_{2}$ molecule using several quantum and classical statistical mechanics methods. The partition functions (and hence the implied thermodynamical properties) calculated from the classical configurational integral and quantum path integral approaches have been shown to diverge at high temperatures. Although the analysis has been limited here to diatomic molecules, the same conclusions should have general validity. They may then be important in computations of thermodynamical properties for larger systems where quantum calculations are out of reach and classical statistical mechanics and/or FPI methods are the only viable routes. ${ }^{2}$ They are also relevant in relation to floppy polyatomic systems such as van der Waals molecules, where classical and quantum results are in good agreement even at low temperatures. ${ }^{13,14,16}$ Indeed, for these systems, the temperature at which the configurational integral based methods (classical and FPI) start to diverge is expected to be only a few hundred Kelvin or so. We should conclude by noting that it is unclear whether the correct prescription for calculating a partition function is always to preclude contributions from dissociative states. It should be so when the focus is on comparisons with partition functions obtained from bound-state eigenvalue calculations or spectroscopic data as in the present work, but it remains an open question whether only genuine bound states should be considered if other types of measurements are to be addressed. Clearly, an answer to this problem is outside the scope of the present work.

Acknowledgment. The authors thank Prof. R.Q. Topper for helpful discussions. This work has the support of Fundação para a Ciência e Tecnologia, Portugal, under Program PRAXIS XXI. It has also benefited from an EC grant under Contract No. CHRX-CT 94-0436. F.V.P. also acknowledges partial financial help through Fundação Coordenação de Aperfeiçoamento de Pessoal de Nivel Superior (CAPES, Brazil).

\section{References and Notes}

(1) Topper, R. Q. Adv. Chem. Phys. 1999, 105, 117.

(2) Srinivsan, J.; Volobuev, Y. L.; Mielke, S. L.; Truhlar, D. G. Comput. Phys. Commun. 2000, 128, 446.

(3) McQuarrie, D. A. Statistical Mechanics; Harper and Row: New York, 1976

(4) Mayer, J. E.; Mayer, M. G. Statistical Mechanics; Wiley: New York, 1963 .

(5) Neale, L.; Tennyson, J. Astrophys. J. 1995, 454, L159.

(6) Harris, G. J.; Viti, S.; Mussa, H. Y.; Tennyson, J. J. Chem. Phys. 1998, 109, 7197.

(7) Feynman, R. P.; Hibbs, A. R. Quantum Mechanics and Path Integrals; McGraw-Hill: New York, 1965. 1972.

(8) Feynman, R. P. Statistical Mechanics; Benjamin: Reading, MA,

(9) Trotter, H. F. Proc. Am. Math. Soc. 1959, 10, 545 401

(11) Schulman, L. S. Techniques and Applications of Path Integration; Wiley-Interscience: New York, 1981.

(12) Chandler, D.; Wolynes, P. G. J. Chem. Phys. 1981, 74, 4078

(13) Dardi, P. S.; Dahler, J. S. J. Chem. Phys. 1990, 93, 3562

(14) Guérin, H. J. Phys. B: At. Mol. Opt. Phys. 1992, 25, 1697.

(15) Riganelli, A.; Wang, W.; Varandas, A. J. C. J. Phys. Chem. A 1999, 103,8303 .

(16) Riganelli, A.; Prudente, F. V.; Varandas, A. J. C. Phys. Chem Chem. Phys. 2000, 2, 4121

(17) Messina, M.; Schenter, G. K.; Garrett, C. J. Chem. Phys. 1993, 98,4120 .

(18) Taubmann, G.; Witschel, W.; Shoendorff, L. J. Phys. B: At. Mol. Opt. Phys. 1999, 32, 2859.

(19) Prudente, F. V.; Riganelli, A.; Varandas, A. J. C. J. Phys. Chem. A 2001, 105, 5272

(20) Hill, T. An Introduction to Statistical Thermodynamics; AddisonWesley: Reading, MA, 1960. 5589 .

(22) Schenter, G. K. J. Chem. Phys. 1998, 108, 6222.

(23) Mielke, S. L.; Srinivasan, J.; Truhlar, D. G. J. Chem. Phys. 2000 $112,8758$.

(24) Bunker, D. L.; Davidson, N. J. Am. Chem. Soc. 1958, 80, 5090.

(25) Abramowitz, M.; Stegun, I. A. Handbook of Mathematical Functions; Dover: New York, 1970.

(26) Irwin, A. W. Astron. Astrophys. 1987, 182, 348.

(27) Varandas, A. J. C.; Silva, J. D. J. Chem. Soc., Faraday Trans. 2 1986, 82, 593.

(28) Varandas, A. J. C.; Silva, J. D. J. Chem. Soc., Faraday Trans. 2 1992, 88, 941 .

(29) Colbert, D. T.; Miller, W. H. J. Chem. Phys. 1992, 96, 1982.

(30) Muckerman, J. T. Chem. Phys. Lett. 1990, 173, 200.

(31) Baye, D.; Heenen, P. H. J. Phys. A: Nath. Gen. 1986, 19, 2041.

(32) Prates Ramalho, J. P.; Silva Fernandes, F. M. Z. Naturforsch. 1990, $45 A, 1193$.

(33) Sethia, A.; Sanyal, S.; Singh, Y. J. Chem. 1990, 93, 7268. 